\title{
Krigagem das proporções utilizando a krigagem da média: uma ferramenta auxiliar na modelagem de reservatórios
}

\author{
Armando Zaupa Remacre $^{1}$, Márcio Nunes Normando ${ }^{2}$ \& Sérgio Sacani Sancevero ${ }^{3}$
}

\begin{abstract}
Resumo As curvas de proporção possuem um importante papel na caracterização geológica de depósitos de hidrocarbonetos. Por meio delas é possível definir, para determinados intervalos, os tipos de fácies dominantes, e essas informações podem posteriormente auxiliar na modelagem do reservatório sendo usadas como dados condicionantes e entendimento de tendências. Porém, ao serem construídas via média aritmética essas curvas não carregam consigo a influência dos poços e a localização dos mesmos. Considerando essas informações, pode-se gerar curvas mais adaptadas a realidade e com informações assim, mais confiáveis. Desse modo, o principal objetivo deste trabalho é mostrar uma metodologia de construção das curvas de proporção utilizando a krigagem da média para estimar as proporções. A vantagem dessa técnica é que a localização dos poços e a possível área de influência dos mesmos pode ser considerada. Com isso, pode-se mostrar como gerar de maneira mais eficiente as curvas de proporções, que uma vez construídas podem auxiliar na caracterização de reservatórios, principalmente no que diz respeito à modelagem litológica.
\end{abstract}

Palavras-Chave: Curvas de proporção, krigagem da média, caracterização de reservatórios, fácies.

\begin{abstract}
Kriging of proportions using mean kriging. The proportion curves have an important role in petroleum geology. With this tool it is possible to define specific intervals for the dominant facies and, as result we can use the proportion curve as a trend in lithological modeling. However, when the proportion curves are generated they don't consider the well influence and the location of the wells. When we consider those information is possible to generate a more effective proportions curves. So, the main aim of this paper is to present a new technique to generate the proportion curves based in the mean kriging. The main advantage of this methodology is that the well location and his influence zone are considered in the process. This methodology indicated a efficient way to generate the proportion curves that can be used to support in a decisive way the reservoir characterization process linking to the lithological modeling.
\end{abstract}

Keywords: Proportion Curves, Mean Kriging, Reservoir Characterization, Facies.

INTRODUÇÃO As curvas de proporção apareceram no final da década de 80 para auxiliar na quantificação dos dados geológicos na caracterização de reservatórios (Ravenne et al., 2002). Quando se estuda um reservatório têm-se parâmetros globais como, por exemplo, 21\% de areia, e "valores locais" como, por exemplo, num bloco, 15\% de areia. Entre essas duas situações, global e local, situam-se as curvas de proporções, como por exemplo, num dado nível ocorre $18 \%$ de areia.

Inicialmente esta ferramenta era utilizada somente para auxiliar no processo de quantificação de dados, em outras palavras, era simplesmente uma ferramenta estatística que auxiliava no condicionamento das simulaçães levando em consideração os dados geológicos (Ravenne, 2002).

As curvas de proporção podem ser vistas como um histograma de fácies, mostrando a ocorrência das mesmas em uma determinada zona do estudo (as mais comuns são as curvas de proporções verticais). Trata-se de uma visualização gráfica da distribuição dos litotipos dentro de uma região, considerando-se a sua freqüência relativa.

As curvas de proporção podem ser calculadas de duas maneiras: vertical e horizontal (Fig. 1). As curvas de proporção vertical (CPV) retratam a proporção média de cada fácies calculada nível a nível, paralelo a um referencial adotado, quantificando uma evolução seqüencial ou temporal das litofácies. Já as curvas de proporção horizontal $(\mathrm{CPH})$ retratam uma proporção acumulada calculada poço a poço, representando a evolução lateral das litofácies. Ambas refletem o comportamento estacionário ou não das fácies.

Apresenta-se na figura 1, de forma esquemática, como são calculadas as curvas de proporção vertical e horizontal de fácies a partir de cinco poços fictícios dispostos em uma malha estratigráfica e com seções testemunhadas de igual comprimento, contendo cinco fácies discretizadas ao longo dos poços.

Pode-se observar na figura 1 que na camada de topo há $60 \%$ de Fácies 5, 20\% de Fácies 4 e 20\% de Fácies 3. Na segunda camada há $20 \%$ de Fácies 5, 60\% de Fácies 4 e 20\% de Fácies 3. Segue-se sucessiva- 


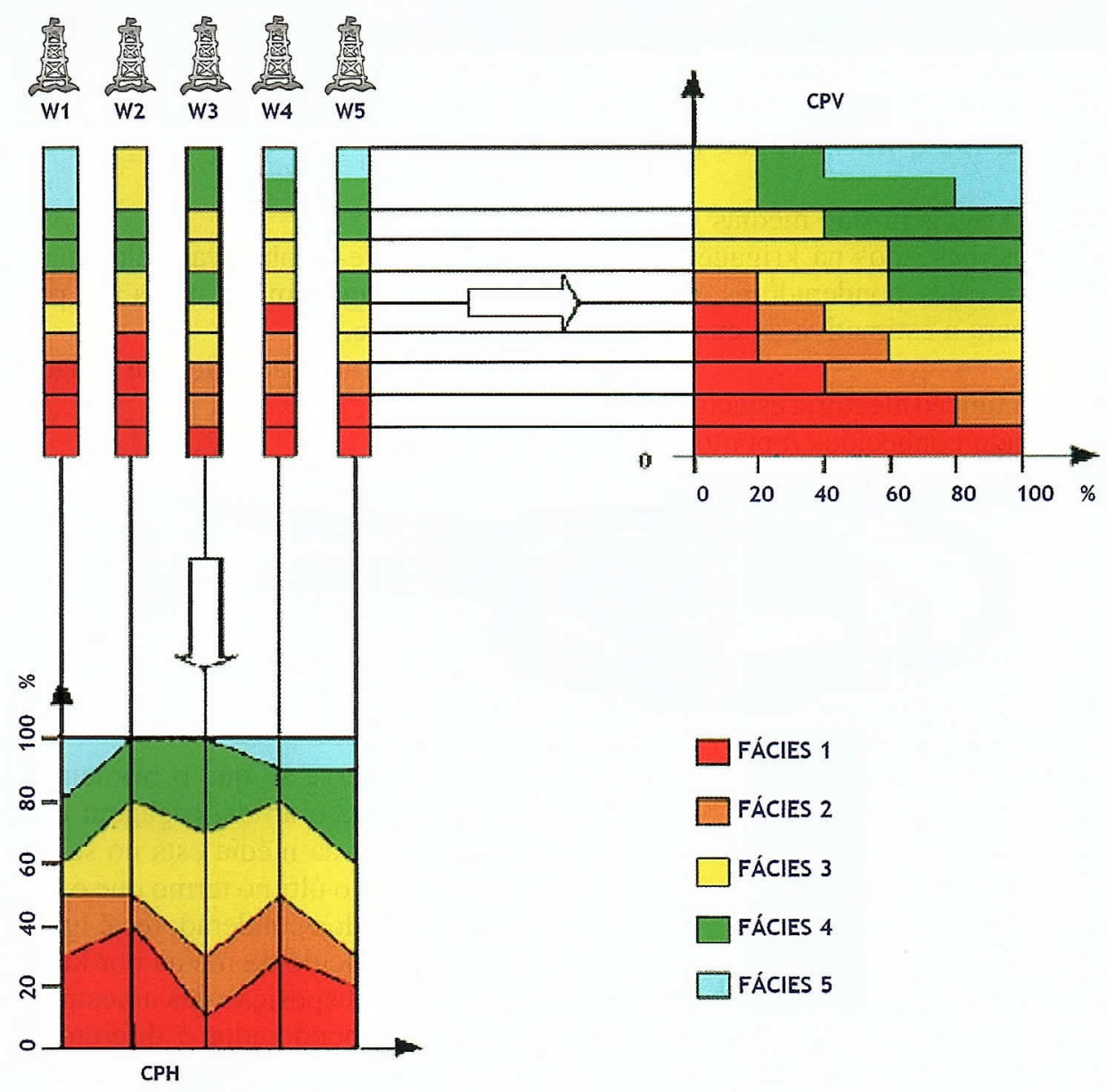

Figura 1 - Curvas de proporção vertical (CPV) e horizontal (CPH) construidas a partir de cinco poços fictícios com cinco fácies (modificado de Souza Jr., 1997).

mente até que na camada basal há $100 \%$ de Fácies 1 . A partir da curva de proporção horizontal não é possível identificar nenhuma localização preferencial de fácies, permitindo que se considere as proporções de fácies estacionárias nessa direção.

Ravenne et al. (2002) citam as curvas de proporção vertical como uma nova ferramenta que auxilia na interpretação de subsuperfícies, ajudando a melhorar na descrição do modelo. Fisicamente estas curvas apresentam a proporção de cada fácies como função da profundidade ou de qualquer outra coordenada. Do ponto de vista geoestatístico estas variações verticais são uma forma de constatar a não estacionariedade. Podem portanto serem usadas para guiar os métodos de simulação estocástica a ser usado, para avaliar os resultados da simulação estocástica e etc.

Esta ferramenta teve sua eficiência demonstrada na validação e coerência da base de dados, na determinação de marcos cronoestratigráficos que são utilizados como níveis de referência servindo para horizontalização antes dos cálculos e, ainda, para correlação entre os poços. Também é utilizada para análise da seqüência estratigráfica que serve para quantificar e visualizar as evoluções laterais e verticais das fácies dentro da seqü- ência deposicional (Ravenne, 2002).

O primeiro passo para o cálculo de uma curva de proporção vertical é escolher um ordenamento de fácies com um significado geológico (por exemplo a granulometria). Depois, calcula-se a proporção experimental e acumulada de cada fácies, nível a nível. Daí obtém-se a curva de proporção vertical, que é um gráfico entre a profundidade versus a proporção acumulada. Em outras palavras, estima-se cada proporção considerando a presença da fácies em cada ponto. Em geral, cada ponto recebe o peso $1 / \mathrm{N}, \mathrm{N}$ sendo o número de pontos (ou poços). Este procedimento é adequado para malhas regulares.

O objetivo deste trabalho é a construção da curva de proporção vertical levando em consideração a influência que cada poço possui em uma determinada área. O que se propõe neste estudo é a utilização da krigagem da média ou, no caso, a krigagem das proporções para a construção da curva de proporção vertical, haja vista que na prática raramente se encontram malhas regulares. Há casos em que existem cluster de dados e esse método, por ser uma krigagem também proporciona a desclusterização. 
MÉTODO A krigagem da média (neste trabalho utiliza-se a denominação krigagem das proporções) foi inicialmente proposta por Matheron em 1970, sendo utilizada por Remacre, 1984, Wackernagel, 1995 e Remacre, 1996.

Por meio da krigagem das médias é possível obter os ponderadores, baseados na krigagem da proporção no nível. Com estes ponderadores, cada poço passa a ter um peso para a estimativa da curva de proporção vertical.

Seja $Z(\mathbf{x})$ uma função aleatória estacionária com variograma $\gamma(\mathbf{h})$ e sendo conhecidos $n$ pontos, pode-se estimar a média das amostras por

$$
m^{*}=\sum_{1}^{n} \lambda_{i} Z\left(\mathbf{x}_{i}\right)
$$

onde $m^{*}$ é a média estimada das amostras, $n$ é o número de amostras, $Z\left(\mathbf{x}_{\mathrm{i}}\right)$ é o valor da amostra e $\lambda_{i}$ é o ponderador. Esses ponderadores devem satisfazer a condição a equação (2):

$$
\sum_{i}^{n} \lambda_{i}=1
$$

Para garantir o não viés da estimativa. Supondo a estacionariedade de $Z(\mathbf{x})$, ou seja, assume-se que a média existe para todos os pontos da região $\mathrm{D}$

$$
\operatorname{var}\left[m^{*}-m\right]=\operatorname{var}\left[m^{*}\right]=-\sum_{i}^{n} \sum_{j}^{n} \lambda_{i} \lambda_{j} \gamma(\boldsymbol{h}),
$$

e o variograma é dado por:

$$
\gamma(\mathbf{h})=\frac{1}{2} E[Z(\boldsymbol{x})-Z(\boldsymbol{x}+\boldsymbol{h})]^{2}
$$

Como a estimativa é uma krigagem, a variância deve ser minimizada

$$
\operatorname{var}\left[m^{*}-m\right]=\operatorname{var}\left[m^{*}\right]=-\sum_{i}^{n} \sum_{j}^{n} \lambda_{i} \lambda_{j} \gamma(\mathbf{h})
$$

As resoluções destas expressões podem ser encontradas em Wackernagel (1995) e Remacre (1996). Ao se minimizar a variância, utiliza-se o multiplicador de Lagrange $(\mu)$ dada a equação 2 . A função a minimizar torna-se

$$
-\sum_{i}^{n} \sum_{j}^{n} \lambda_{i} \lambda_{j} \gamma(\mathbf{h})-2 \mu\left(\sum_{i}^{n} \lambda_{i}-1\right)
$$

que derivando em relação a $\lambda$ e igualando a zero, resulta no sistema:

$$
\begin{aligned}
& \sum_{j}^{n} \lambda_{j} \gamma(\mathbf{h})-\mu=0 \quad i=1,2, \ldots, n \\
& \sum_{i}^{n} \lambda_{i}=1
\end{aligned}
$$

Resolvendo-se este sistema, a variância mínima torna-se:

$$
\operatorname{var}\left[m^{*}\right]=-\sum_{i}^{n} \sum_{j}^{n} \lambda_{i} \lambda_{j} \gamma(\mathbf{h})=\mu
$$

Pode-se observar pela equação (8) que a variância da krigagem da média é o próprio multiplicador de Lagrange.

Em notação matricial o sistema (9) fica:

$$
\left(\begin{array}{ccccc}
\gamma_{11} & \gamma_{12} & \cdots & \gamma_{1 n} & 1 \\
\gamma_{21} & \gamma_{22} & \cdots & \gamma_{2 n} 1 \\
\vdots & \vdots & \ddots & \vdots & \\
1 & 1 & & & 0
\end{array}\right)\left(\begin{array}{c}
\lambda_{1} \\
\lambda_{2} \\
\vdots \\
\mu
\end{array}\right)=\left(\begin{array}{c}
0 \\
0 \\
\vdots \\
1
\end{array}\right)
$$

onde $\gamma_{i j}$ é o variograma estacionário entre as amostras $i$ $\mathrm{e} j$.

Observa-se que o primeiro membro deste sistema é idêntico ao da krigagem ordinária, a diferença na krigagem da média está no segundo membro que é nulo, exceto o último termo que é um, devido ao fato de que a soma dos ponderadores é igual a um.

O cálculo da média por krigagem leva em consideração a disposição das amostras no espaço, ou seja, o valor do ponderador é diferente para cada amostra dependendo da disposição espacial e do modelo de correlação que é assumido existir entre as amostras. Desta forma, os dados na borda têm ponderadores diferentes daqueles no centro e os dados agrupados têm um valor de ponderador diferente daqueles isolados.

RESULTADOS E DISCUSSÃO Para demonstrar a metodologia proposta neste trabalho utilizaram-se cinco poços verticais dispostos em malha irregular que atravessam 5 níveis e apresentam 3 fácies (Fácies 1 , Fácies 2 e Fácies 3). Assumiu-se também que todos os poços estão referenciados a um mesmo horizonte (Fig. 3). Calcularam-se também as médias aritméticas nesses 5 níveis para mostrar a diferença na curva de proporção vertical construída pelo método tradicional (proporção média de nível) e pelo método proposto (krigagem das médias). Na figura 2 apresenta-se o mapa de localização dos cinco poços usados neste estudo.

A curva de proporção vertical da área constituída por estes poços, baseada no método de proporção média dos poços é mostrada na figura 4 , onde se observa que no nível zero há $20 \%$ de Fácies 1 e $80 \%$ de Fácies 2, no nível 1 há $20 \%$ de Fácies 1, 60\% de Fácies 2 e $20 \%$ de Fácies 3, no nível 2 há $20 \%$ de Fácies 1 , $40 \%$ de Fácies 2 e $40 \%$ de Fácies 3, no nível 3 há 20\% de Fácies 1, 40\% de Fácies 2 e 40\% de Fácies 3 e, por fim, no nível 4 há $20 \%$ de Fácies 1, 20\% de Fácies 2 e $60 \%$ de Fácies 3 .

Analisando este caso, percebe-se que a Fácies 1 está presente em todos os poços com a mesma proporção. Como são cinco poços, a Fácies 1 assume uma proporção de $20 \%$ para cada nível de profundidade. 


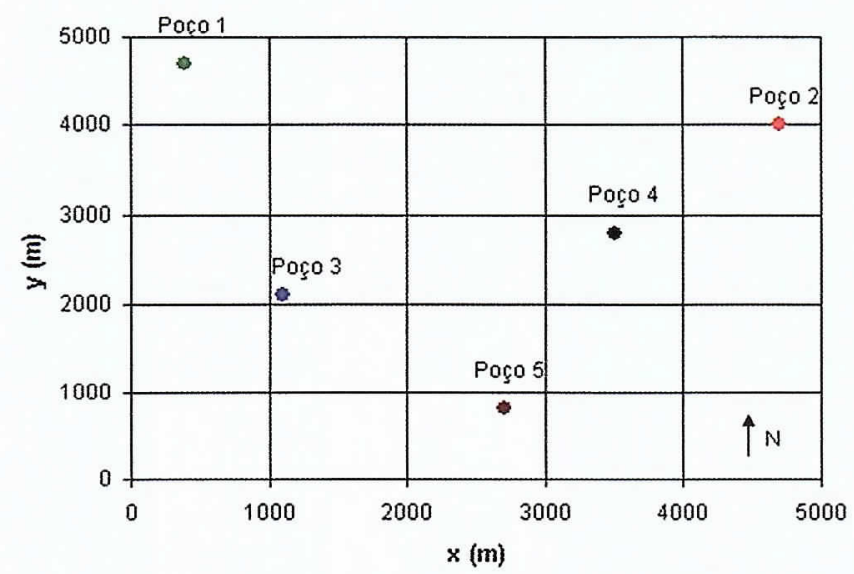

Figura 2 - Mapa de locação dos 5 poços usados neste estudo.

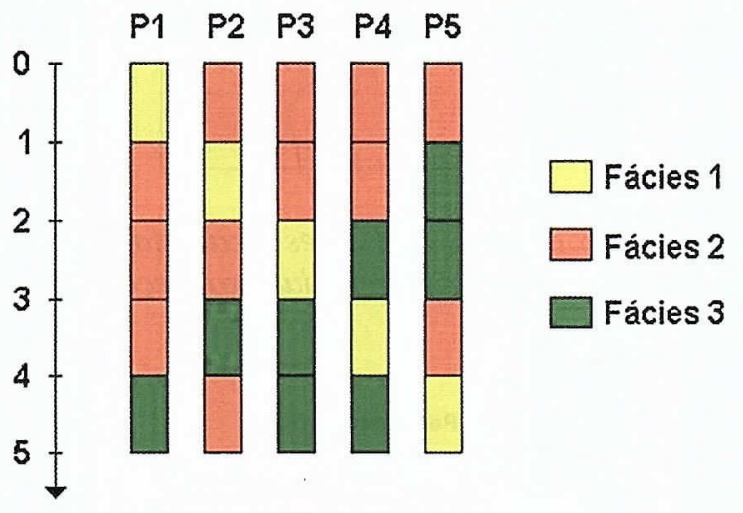

Figura 3 - Representação dos cinco poços deste estudo e suas respectivas fácies relacionadas aos niveis de profundidade.

Observando o mapa base, da localização dos poços, pode-se observar que cada poço possui uma área de influência e esta varia de poço para poço. Deste modo utilizou-se a krigagem das médias para obter os valores dos ponderadores que levam em consideração a disposição e correlação entre os poços.

Para se calcular os ponderadores por meio da

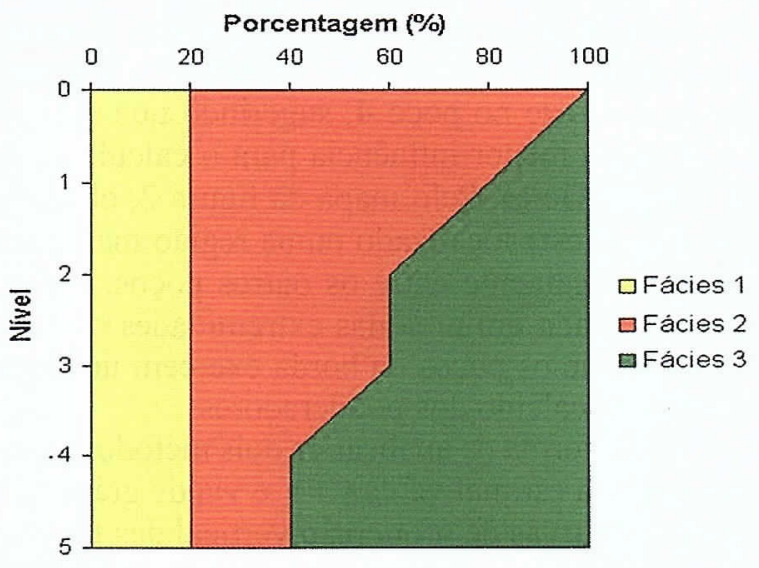

Figura 4 - Curva de proporção vertical baseada na proporção média dos poços.

krigagem da média e deste modo se estimar as proporções, utilizou-se um mesmo modelo de variograma para todos os níveis. Para a indicatriz das Fácies 1 e 2 utilizou-se um modelo de variograma esférico com alcance de $4000 \mathrm{~m}$, já para a indicatriz da Fácies 3 foi usado um modelo de variograma esférico com alcance de $6000 \mathrm{~m}$. A partir da indicatriz de cada fácies obtiveram-se os valores de suas proporções para cada nível, por krigagem.

Na tabela 1 são apresentados os resultados dos cálculos das proporções de cada fácies para os níveis de profundidade por meio da krigagem da média e pela média aritmética.

Observa-se na tabela 1 que os valores das proporções calculados por krigagem das médias são diferentes dos calculados por média aritmética pelo fato da krigagem levar em consideração a posição dos poços. Nota-se, por exemplo, que o valor da proporção para a Fácies 1 por média aritmética é igual a $20 \%$ para todos os níveis, já o valor por krigagem das médias varia em cada nível por levar em conta a disposição dos poços na área em estudo.

Para a Fácies 1, observa-se que o nível zero é onde se apresenta a maior influência desta fácies e corresponde ao poço 1 , o que significa dizer que o poço 1 é o que tem a maior influência para esta fácies no cálcu-

Tabela 1 - Valores das proporções das fácies calculados pela média aritmética e por krigagem das médias.

\begin{tabular}{l|c|c|c|c|c|c}
\hline \multirow{2}{*}{ Profundidade } & \multicolumn{7}{|c|}{ Valor das proporções das fácies } \\
\cline { 2 - 7 } & \multicolumn{2}{|c}{ Fácies 1 } & \multicolumn{2}{c}{ Fácies 2 } & \multicolumn{2}{c}{ Fácies 3 } \\
\cline { 2 - 7 } & $\begin{array}{c}\text { Por média } \\
\text { aritmética }\end{array}$ & $\begin{array}{c}\text { Por krigagem } \\
\text { das médias }\end{array}$ & $\begin{array}{c}\text { Por média } \\
\text { aritmética }\end{array}$ & $\begin{array}{c}\text { Por krigagem } \\
\text { das médias }\end{array}$ & $\begin{array}{c}\text { Por média } \\
\text { aritmética }\end{array}$ & $\begin{array}{c}\text { Por krigagem } \\
\text { das médias }\end{array}$ \\
\hline Nível 0 & 0,200 & 0,239 & 0,800 & 0,761 & 0,000 & 0,000 \\
\hline Nível 1 & 0,200 & 0,193 & 0,600 & 0,575 & 0,200 & 0,232 \\
\hline Nível 2 & 0,200 & 0,233 & 0,400 & 0,534 & 0,400 & 0,233 \\
\hline Nível 3 & 0,200 & 0,115 & 0,400 & 0,405 & 0,400 & 0,480 \\
\hline Nível 4 & 0,200 & 0,220 & 0,200 & 0,259 & 0,600 & 0,521 \\
\hline
\end{tabular}


lo da curva de proporção vertical. Observa-se também que no nível 3, que tem o menor valor da proporção, a Fácies 1 ocorre no poço 4, sugerindo que este poço é o que tem a menor influência para o cálculo da proporção desta fácies. Pelo mapa da figura 2, observa-se que o poço 4 está localizado numa região mais central da área, praticamente entre os outros poços, e o poço 1 está localizado em uma das extremidades da área, o que sugere que os poços da borda exercem uma maior influência no cálculo dos ponderadores.

Uma forma de analisar os dois métodos aqui estudados para a estimativa da CPV é via de gráficos que mostram as curvas de proporção vertical das fácies. As Figuras 5, 6 e 7 mostram as curvas de proporção vertical das Fácies 1, 2 e 3, respectivamente, para os cinco poços em estudo, calculadas a partir da média aritmética e krigagem das proporções.

Na figura 5 observa-se que enquanto a média aritmética produz uma CPV constante para a Fácies 1, a CPV construída por krigagem das proporções não é constante e seus valores, para os níveis de profundidade, dependem da posição dos poços. Como se adotou o mesmo variograma para todos os níveis esses ponderadores são iguais para a facies 1 e assim por diante.

Observa-se na figura 6 que as proporções da Fácies 2 também diferem quando se calcula o valor por krigagem das proporções. Nota-se que no nível 2 a di- ferença entre os valores das proporções é maior e nos outros níveis os valores estão mais próximos.

Na figura 7 percebe-se uma maior diferença nos valores das proporções também no nível 2 e diferenças razoáveis nos outros níveis. O importante é observar que houve uma diferença entre no valor das proporções das fácies utilizando os dois métodos para a estimativa da CPV. A figura 8 representa as curvas de proporção vertical calculadas por proporção média dos poços e

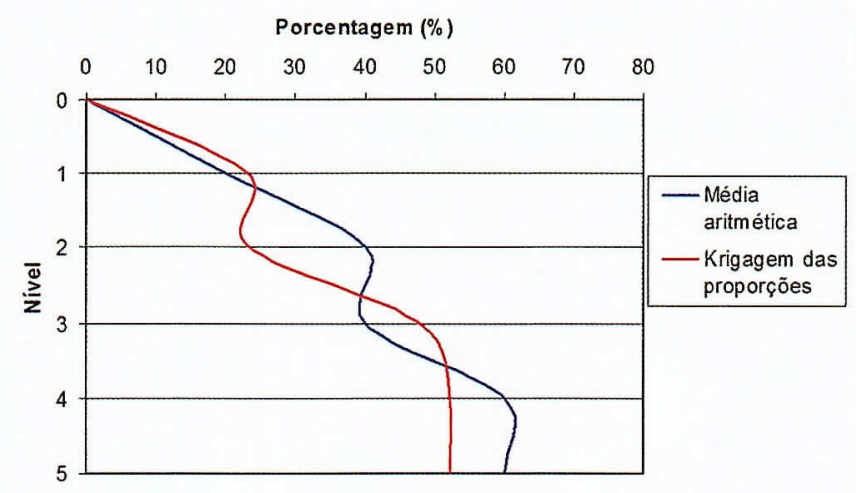

Figura 7 - Proporções da Fácies 3 calculadas por média aritmética e por krigagem das proporções.

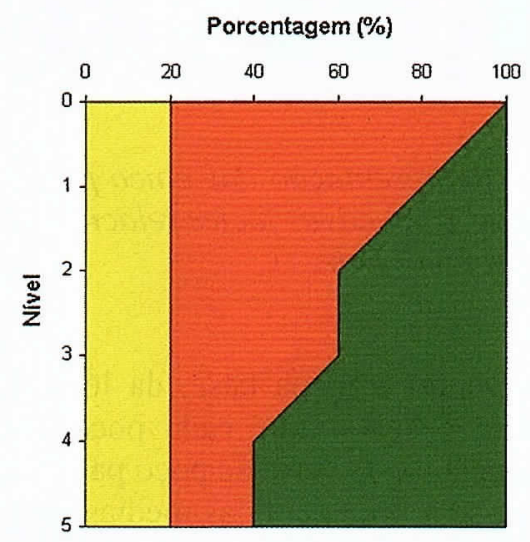

(a)

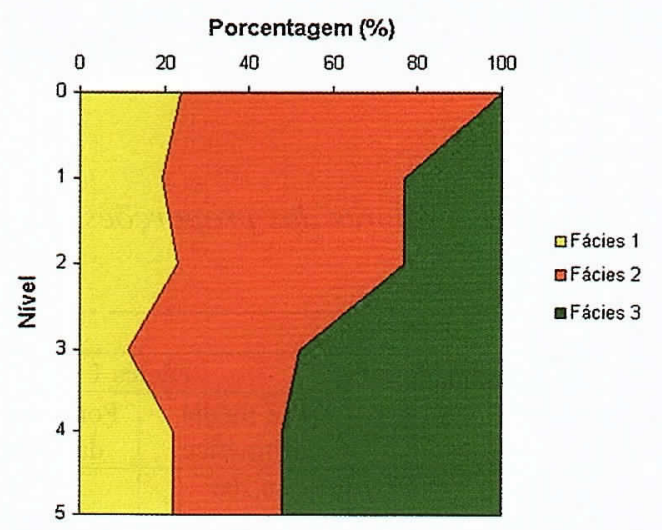

(b)

Figura 8 - CPV calculada por (a) proporção média dos poços e (b) por krigagem das proporções.
Figura 6 - Proporções da Fácies 2 calculadas por média aritmética e por krigagem das proporções.
Figura 5 - Proporções da Fácies 1 calculadas por média aritmética e por krigagem das proporções.

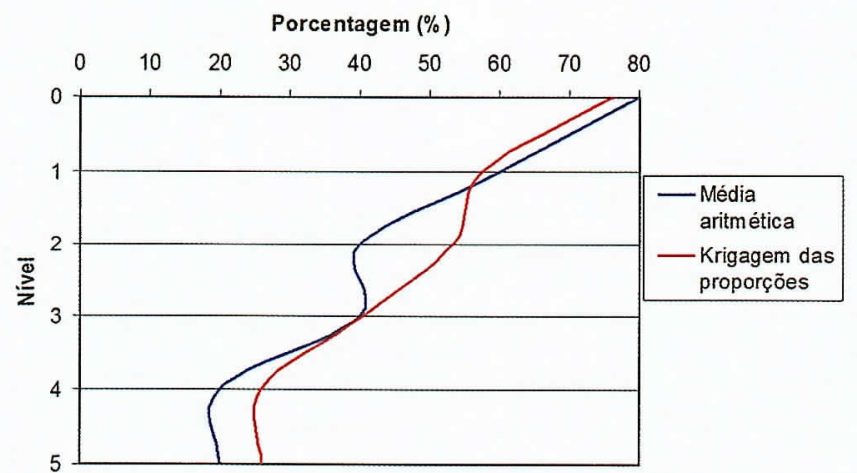


por krigagem das proporções.

Pode-se observar que existem diferenças entre os valores das proporções das fácies nas curvas de proporção vertical mostradas na figura 8 . Na CPV calculada por krigagem das médias não se obteve uma porcentagem de Fácies 1 constante, como no caso da CPV por proporção média dos poços, mas ambas mostraram seguir uma mesma tendência.

CONCLUSÕES Neste trabalho calculou-se a curva de proporção vertical para uma área com cinco poços e três fácies usando de dois métodos distintos. O primeiro foi baseado na proporção média dos poços sendo a abordagem utilizada rotineiramente para a construção de curvas de proporção vertical. O segundo método foi baseado na krigagem das proporções e, difere do primeiro, pelo fato de levar em consideração, para o cálculo da CPV, a influência que cada poço tem na área em estudo devido a sua disposição espacial com relação aos demais poços.

Realizou-se neste estudo um caso simples com poços verticais e admitindo-se um modelo idêntico de variograma para todos os níveis. Entretanto, na prática podem ser observados diversos níveis de anisotropias que ocorrem em diferentes profundidades, obtendo-se assim distintos ponderadores.

No caso de poços inclinados deve-se fazer um estudo geoestatístico preliminar para cada nível e determinar, se for o caso, o modelo variográfico por nível. De qualquer modo se os poços são inclinados, tem-se para cada nível um mapa base diferente e, conseqüentemente, ponderadores diferentes. A experiência com a krigagem da média (krigagem das proporções) tem demonstrado que os poços situados nas bordas, por representarem maiores áreas terão maiores pesos.

Os resultados indicam marcantes diferenças en- tre as proporções de fácies obtidas por intermédio dos dois métodos utilizados neste trabalho. Desse modo, a escolha do método de determinação da proporção vertical de fácies é uma tarefa que deve ser realizada de maneira cautelosa, pois irá afetar de maneira decisiva a geração de modelos para a caracterização de reservatórios.

Finalmente, tanto na estimativa de reservas como na simulação estocástica o condicionamento realizado por meio das curvas de proporção vertical terá influência importante na geração dos modelos de reservatório. A krigagem por ser um método que realiza o desagrupamento dos dados (desclustering) já considera a krigagem das proporções implicitamente, mas a simulação estocástica usa diretamente estas curvas. A krigagem da média fornece ponderadores maiores as amostras da borda da vizinhança, fornecendo, às vezes, estimativas pessimistas dessas proporções. Usar diretamente as médias aritméticas pode não ser uma solução adequada, pois não se sabe se estamos sendo otimistas ou pessimista

A escolha do método para a definição das CPV's depende do grau de conhecimento geológico sobre o reservatório a ser modelado. A geração do modelo litológico é determinante para a caracterização de reservatórios, pois a partir dele as propriedades petrofísicas são modeladas, bem como a correta definição das fácies e suas proporções tem influência em todo o processo de caracterização de reservatórios. Em determinadas situações em reservatórios que apresentam elevada heterogeneidade considerar a localização e a relação existente entre os poços, no caso de se utilizar a krigagem da média para se estimar as proporções, pode gerar modelos mais confiáveis para a caracterização de reservatórios.

Agradecimentos À CAPES, pela bolsa de mestrado.

\section{Referências}

Matheron G. 1970. La théorie des variables regionalisées et ses applications, les cahiers du Centre de Geostatistique et Morphologie Mathématique. Fontainebleau, École de Mines de Paris, vol.5.

Ravenne C. 2002. Stratigraphy and oil: a review. Part 2: Characterization of reservoirs and sequence stratigraphy: quantification and modeling. Oil and Gas Science and Technology, 57(4):311-340,

Ravenne C., Galli A., Doligez B., Beucher H., Eschard R. 2002. Quantification of facies relationships via proportion curves. In: Armstrong M., Bettini C., Champigny N., Galli A. (eds.) Geostatistics Rio 2000. Dordrecht, Kluwer Academic Press, p. 19-39.

Remacre A. Z. 1984. L'estimation du récupérable local: le conditionnement uniforme. Thèse Doct. Ing. en Sciences et Techniques Minières, École de Mines Paris, 99p.

Remacre A.Z. 1996. Krigagem da média. Geociências (São Paulo), 15(1):9-16,

Souza Jr. O.G. 1997. Stratigraphie séquentielle et modélisation probabiliste des reservoirs d'un cone sous-marin profound (Champ de Namorado, Brèsil). $\mathrm{PhD}$ thesis Université Paris, Paris, 215p.

Wackernagel H. 1995. Multivariate Geostatistics. Berlin, Springer Verlag, 256p.

Manuscrito BR 05

Submetido em 21 de dezembro de 2007 Aceito em 21 de maio de 2008 